\title{
Examination of Ureaplasma urealyticum and Mycoplasma hominis in 4082 Chinese patients
}

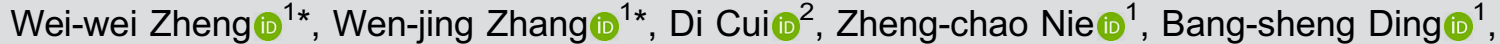 \\ Jiang-hua Cheng (10 ${ }^{3}$, and Chuan-zhong Mei $\mathbb{1}^{2}$
}

${ }^{1}$ Division of Life Sciences and Medicine, Department of Clinical Laboratory, First Affiliated Hospital of University of Science and Technology of China, Hefei, Anhui, China

${ }^{2}$ School of Laboratory Medicine, Bengbu Medical College, Bengbu, Anhui, China ${ }^{3}$ Institute of Agro-products Processing Research, Anhui Academy of Agricultural Sciences, Hefei, Anhui, China

\begin{abstract}
The objective of this study was to analyze the infection rate and drug resistance of Ureaplasma urealyticum (UU) and Mycoplasma hominis (MH) in the genitourinary tract of Chinese patients. From December 2018 to June 2019, vaginal secretion or urinary secretion of outpatients in our hospital were selected for culture and drug sensitivity analysis of Ureaplasma urealyticum and Mycoplasma hominis. In 4082 Chinese samples, 1567 Mycoplasma were detected, a detection rate of 38.39\%, among which 1366 cases were UU single positive, accounting for $33.47 \%, 15$ cases were $\mathrm{MH}$ single positive, accounting for $0.36 \%, 186$ cases were $\mathrm{UU}$ and $\mathrm{MH}$ mixed positive, accounting for $4.56 \%$. The most affected age groups were $21-30$ years and 31-40 years, accounting for 19.09 and $15.05 \%$, respectively. The results of drug sensitivity showed that doxycycline, minocycline, josamycin, clarithromycin, and roxithromycin were more sensitive to mycoplasma infection. The distribution of Ureaplasma urealyticum and Mycoplasma hominis in the human genitourinary system and their sensitivity to antibiotics is different for sex and age groups.
\end{abstract}

Key words: Ureaplasma urealyticum; Mycoplasma hominis; Drug sensitivity

\section{Introduction}

Ureaplasma urealyticum (UU) and Mycoplasma hominis $(\mathrm{MH})$ are the most common Mycoplasma bacteria types, which are one of the main causes of male non-gonococcal urethritis, female cervical infection, abortion, and other diseases $(1,2)$. In recent years, with the abuse of antibiotics, non-standard drug use, repeated infection, and other factors, the drug resistance of $\mathrm{UU}$ and $\mathrm{MH}$ has increased, which brings many difficulties to a standardized clinical treatment $(3,4)$. In order to determine the infection rate and drug resistance of Ureaplasma urealyticum and Mycoplasma hominis in China, we examined the culture and drug sensitivity in 4082 cases of urogenital tract infection.

\section{Material and Methods}

\section{General data}

A total of 4082 patients with urogenital tract infection were selected from the Departments of Urology, Gynecology, Obstetrics, Reproductive Center, and
Dermatovenereology of the First Affiliated Hospital of USTC from December 2018 to July 2019, including 1458 males and 2624 females, aged $34.53 \pm 12.42$ years. The patients were initially diagnosed and did not use antibiotics within two weeks before sample collection. The study was approved by the Ethics Committee of the First Affiliated Hospital of USTC, and informed consent was signed by patients participating in the study.

\section{Specimen collection}

For male patients, a sterile cotton ball was used to clean the urinary meatus, and a sterile cotton swab was then inserted with rotation into the urinary meatus for $1-2 \mathrm{~cm}$ to collect urethral secretion for immediate examination. For female patients, a sterile cotton swab was used to wipe the mucus on the cervical surface. Then, a special swab was inserted for 1-2 cm with rotation several times and remained for $15 \mathrm{~s}$ to collect a sample for examination.

Correspondence: Chuan-zhong Mei: <meichzh@sina.com> | Jiang-hua Cheng: <elmcheng@aaas.org.cn>

*These authors contributed equally to this work.

Received May 23, 2020 | Accepted September 16, 2020 


\section{Culture, identification, and drug sensitivity of Mycoplasma}

Mycoplasma IC kit produced by Zhuhai Lizhu Reagent Co., Ltd. (China) was used. All the reagents were used within the validity period. Drugs tested for sensitivity included: doxycycline, minocycline, josamycin, clarithromycin, roxithromycin, azithromycin, ofloxacin, levofloxacin, and sparfloxacin. High and low concentrations of each antibiotic were used simultaneously. The diluent was added to the $2.9-\mathrm{mL}$ mark of the dry powder bottle of the culture medium, shaken well, and $50 \mu \mathrm{L}$ was transferred to hole $C$ after the dry powder was completely dissolved as the negative control. The swab was put into the culture solution, squeezed, and rotated to completely dissolve the sample. Then, $50 \mu \mathrm{L}$ of the culture solution was placed into each hole, and 1-2 drops of liquid paraffin was placed into the remaining holes. The samples were incubated at $35-37^{\circ} \mathrm{C}$ for 24 or $48 \mathrm{~h}$. UU results were observed after $24 \mathrm{~h}$ and $\mathrm{MH}$ results after $48 \mathrm{~h}$. If the color of the culture hole changed from yellow to red, this indicated that there was Mycoplasma growth $(+)$, if there was no color change (yellow), this indicated no Mycoplasma growth (-). The drug sensitivity was checked as follows: when the indicator hole indicated that there was growth of UU or $\mathrm{MH}$ and the high and low drug concentration pores did not turn red, the drug was considered sensitive (S); when the low concentration hole turned red and the high concentration hole remained unchanged, it was considered intermediary (I); when the high and low concentration holes turned red, it was considered resistant $(R)$.

The SPSS 20.0 software (IBM, USA) was used for statistical analysis. Continuous variables of normal distribution are reported as means $\pm S D$, and the comparison between two groups was done with the $t$-test. Continuous variables of non-normal distribution are reported as median (interquartile range), and the comparison between the two groups was done with a non-parameter test. The counting data are reported as percentage (\%), and chi-squared test was used for comparison between the two groups. The significance level was $\alpha=0.05$.

\section{Results}

\section{Infection rate of Mycoplasma}

There were both single infections and mixed infections in the 4082 Chinese patients. The single infection rate of UU was $33.47 \%$ and of $\mathrm{MH}$ was $0.36 \%$, and the mixed infection rate of $\mathrm{UU}+\mathrm{MH}$ was $4.56 \%$. The infection rate of Mycoplasma in females was $48.21 \%$, among which the infection rates of $\mathrm{UU}, \mathrm{MH}$, and $\mathrm{UU}+\mathrm{MH}$ were $41.65,0.38$, and $6.17 \%$, respectively, and that of Mycoplasma in males was $20.71 \%$, among which the infection rates of $\mathrm{UU}, \mathrm{MH}$, and $\mathrm{UU}+\mathrm{MH}$ were $18.72,0.34$, and $1.65 \%$, respectively. The infection rate of Mycoplasma and of UU infection in females was significantly higher than that in males $(P<0.001)$. There was no significant difference between sexes for $\mathrm{MH}$ infection $(\mathrm{P}=0.847)$. The mixed infection rate of $\mathrm{UU}+\mathrm{MH}$ in women was higher than that in men $(P<0.001)$. See Table 1 for details.

\section{Comparison of Mycoplasma detection in different age groups}

There were 19 positive patients under 20 years old, with a detection rate of $0.48 \%$; 779 positive patients (19.09\%) between 21 and 30 years old; 615 positive patients (15.05\%) between 31 and 40 years old, with a detection rate of $15.05 \%$; 121 positive patients $(2.96 \%)$ between 41 and 50 years old; and 33 positive patients $(0.81 \%)$ over 50 years old. The total detection rate of each age group was the highest in the 21-30 and 31-40 age groups. The detection rate of the 21-30 age group was significantly higher than that of the $\leqslant 20,41-50$, and $>50$ age groups, 31-40 age group was higher than that of $\leqslant 20,41-50$, and $>50$ age groups, and there was no significant difference between 21-30 age group and $31-40$ age group $\left(\chi^{2}=1.591, P=0.207\right)$. See Supplementary Table S1 for details.

\section{Sensitivity results of nine drugs}

The top three drug sensitivity rates of 1366 single UUpositive samples were josamycin (99.63\%), minocycline $(97.52 \%)$, and qianglimycin $(97.29 \%)$; the top three drug resistance rates were ofloxacin $(19.91 \%)$, levofloxacin $(18.23 \%)$, and azithromycin $(9.44 \%)$. The top three drug

Table 1. Detection rate of Mycoplasma in 4082 Chinese samples.

\begin{tabular}{|c|c|c|c|c|c|}
\hline Gender & Number & $\mathrm{UU}(+) \mathrm{MH}(-)$ & $\mathrm{UU}(-) \mathrm{MH}(+)$ & $\mathrm{UU}(+) \mathrm{MH}(+)$ & Total \\
\hline Male & $1458(35.72)$ & $273(18.72)$ & $5(0.34)$ & $24(1.65)$ & $302(20.71)$ \\
\hline Female & 2624 (64.28) & $1093(26.77)$ & $10(0.25)$ & $162(3.97)$ & 1265 (30.99) \\
\hline Total & $4082(100.00)$ & $1366(33.47)$ & $15(0.36)$ & $186(4.56)$ & 1567 (38.39) \\
\hline$\chi^{2}$ value & & 734.682 & 0.037 & 44.179 & 299.579 \\
\hline$P$ value & & $<0.001$ & 0.8475 & $<0.001$ & $<0.001$ \\
\hline
\end{tabular}

Data are reported as number and percent within parentheses. The chi-squared test was used for statistical analysis. UU: Ureaplasma urealyticum; MH: Mycoplasma hominis. 
Table 2. Sensitivity to nine drugs of either/or Ureaplasma urealyticum and Mycoplasma hominis.

\begin{tabular}{lccc}
\hline Drug & Sensitive (n, \%) & Resistant (n, \%) & Intermediate (n, \%) \\
\hline Doxycycline & $1518(96.88)$ & $34(2.17)$ & $7(0.45)$ \\
Minocycline & $1522(97.13)$ & $24(1.53)$ & $21(1.34)$ \\
Josamycin & $1549(98.85)$ & $11(0.70)$ & $7(0.45)$ \\
Clarithromycin & $989(63.12)$ & $250(15.95)$ & $328(20.93)$ \\
Roxithromycin & $1325(84.56)$ & $203(12.95)$ & $39(2.49)$ \\
Azithromycin & $334(21.31)$ & $329(21.00)$ & $904(57.69)$ \\
Ofloxacin & $295(18.83)$ & $414(26.42)$ & $858(54.75)$ \\
Levofloxacin & $122(7.79)$ & $414(26.42)$ & $1031(65.79)$ \\
Sparfloxacin & $231(14.74)$ & $214(13.66)$ & $1122(71.60)$ \\
Total & $1567(100.00)$ & & \\
\hline
\end{tabular}

sensitivity rates of 15 single $\mathrm{MH}$-positive samples were doxycycline $(100 \%)$, minocycline $(100 \%)$, and josamycin $(100 \%)$; the top three drug resistance rates were clarithromycin $(100 \%)$, roxithromycin $(100 \%)$, and azithromycin $(100 \%)$. The top three drug sensitivity rates of $186 \mathrm{UU}$ - and $\mathrm{MH}$-positive samples were minocycline (94\%) doxycycline (93.55\%), and josamycin (93.01\%); the top three drug resistance rates were azithromycin $(99.46 \%)$, clarithromycin $(98.92 \%)$, and roxithromycin $(97.31 \%)$. After summarizing the drug resistance of all 1567 Mycoplasma positive samples, we found that the sensitivity of doxycycline, minocycline, and josamycin exceeded $95 \%$, while the drug resistance rate of ofloxacin, levofloxacin, and azithromycin exceeded 20\%. See Supplementary Table S2 and Table 2 for details.

\section{Discussion}

The Mycoplasma that can cause reproductive system infection mainly includes Ureaplasma urealyticum and Mycoplasma hominis. At present, these two Mycoplasma can cause pyelonephritis, vaginitis, pelvic inflammation, and other diseases, affecting the quality of life of patients. Therefore, once diagnosed, we must give timely and standardized treatment (5). The results of this study showed that the total infection rate of Mycoplasma in 4082 Chinese patients was $38.39 \%$, mainly due to UU single infection, which was not consistent with the results of some research (6-8), indicating some differences in the distribution of Mycoplasma in different areas, which may be due to the use of antibiotics, sampling sites, detection reagents, and laboratory precision level in different areas.

The rate of Mycoplasma infection was $48.21 \%$ in women and $20.71 \%$ in men. The result is different from other areas in China and other countries (9-11). This may be due to the physiology of women, which is more favorable to infection by Mycoplasma. Another reason could be the number of infertility patients or women with other problems in the reproductive center, which has increased in recent years.

The higher infection rate in the 21-30-year and 31-40-year groups may be due to the fact that these age groups are in the childbearing period and have active sexual lives. Some patients had multiple sexual partners or other pathogens such as Neisseria gonorrhoeae and Chlamydia, and the vast majority of Mycoplasma infections are sexually transmitted. A promiscuous sexual life would also increase the probability of infection (12).

Mycoplasma have no cell wall and are naturally resistant to antibiotics acting on the cell wall such as $\beta$-lactams. Therefore, antibiotics acting on nucleoprotein should be selected to achieve effective treatment. In this study, doxycycline and minocycline were selected as tetracycline antibiotics, josamycin, clarithromycin, roxithromycin, and azithromycin as macrolides, and ofloxacin, levofloxacin, and sparfloxacin as quinolones. Our results showed that UU infection was sensitive to most antibiotics, such as josamycin, minocycline, doxycycline, and clarithromycin, and the drug resistance rate was low. $\mathrm{MH}$ infection and $\mathrm{UU}+\mathrm{MH}$ mixed infection were resistant to clarithromycin, roxithromycin, azithromycin, ofloxacin, and levofloxacin. This is because $\mathrm{MH}$ has multiple drug resistance genes, natural resistance to most macrocyclic ester drugs, and cross-resistance to quinolones (13). In this study, the sensitivities of doxycycline, minocycline, and josamycin were over $95 \%$, while the resistance of ofloxacin, levofloxacin, and azithromycin were over $20 \%$, which indicated that appropriate antibiotics should be selected for different types of Mycoplasma.

In summary, clinicians should carry out routine Mycoplasma culture and identification and drug sensitivity tests to select appropriate antibiotics according to the results and achieve the best treatment effect.

\section{Supplementary Material}

Click here to view [pdf].

\section{Acknowledgments}

This study was supported by the National Natural Scientific Foundation of China (No. 81700154), China Postdoctoral Science Foundation (2019M662209), Research Fund for Postdoctoral Researchers of Anhui Province (2019B380), and Teaching Research Project of Anhui Education Department (2017jyxm1096). 


\section{References}

1. Moragianni D, Dryllis G, Andromidas $P$, Kapeta-Korkouli R, Kouskouni E, G, Andromidas P, et al. Genital tract infection and associated factors affect the reproductive outcome in fertile females and females undergoing in vitro fertilization. Biomed Rep 2019; 10: 231-237, doi: 10.3892/br.2019.1194.

2. Andrzejczak-Grzadko S, Dolińska I. Detection of urogenital mycoplasmas in clinical samples [in Polish]. Postepy Biochem 2019; 65: 299-305, doi: 10.18388/pb.2019_273.

3. Gu X, Liu S, Guo X, Weng R, Zhong Z. Epidemiological investigation and antimicrobial susceptibility analysis of mycoplasma in patients with genital manifestations. J Clin Lab Anal 2020; 3: e23118.

4. Ahmadi $\mathrm{MH}$, Mirsalehian A, Bahador A. Prevalence of urogenital mycoplasmas in Iran and their effects on fertility potential: a systematic review and meta-analysis. Iran $J$ Public Health 2016; 45: 409-422.

5. Yang T, Pan L, Wu N, Wang L, Zhen Liu, Kong $\mathrm{Y}$, et al. Antimicrobial resistance in clinical Ureaplasma spp. and Mycoplasma hominis and structural mechanisms underlying quinolone resistance. Antimicrob Agents Chemother 2020; 64: e02560-19, doi: 10.1128/AAC.02560-19.

6. Ling Qing, Qi-Xiang Song, Jian-Li Feng, Hai-Yan Li, Guiming Liu, Hai-Hong Jiang. Prevalence of Chlamydia trachomatis, Neisseria gonorrhoeae, Mycoplasma genitalium and Ureaplasma urealyticum infections using a novel isothermal simultaneous RNA amplification testing method in infertile males. Ann Clin Microbiol Antimicrob 2017; 16: 45, doi: 10.1186/s12941-017-0220-2.

7. Leli C, Mencacci A, Latino MA, Clerici P, Rassu M, Perito S, et al. Prevalence of cervical colonization by Ureaplasma parvum, Ureaplasma urealyticum, Mycoplasma hominis and Mycoplasma genitalium in childbearing age women by a commercially available multiplex real-time PCR: An Italian observational multicentre study. J Microbiol Immunol Infect 2018; 51: 220-225, doi: 10.1016/j.jmii.2017.05.004.

8. Skiljevic D, Mirkov D, Vukicevic J. Prevalence and antibiotic susceptibility of Mycoplasma hominis and Ureaplasma urealyticum in genital samples collected over 6 years at a Serbian university hospital. Indian J Dermatol Venereol Leprol 2016; 82: 37-41, doi: 10.4103/03786323.172903.

9. Diadhiou M, Diallo AB, Barry MS, Alavo SC, Mall I, Gassama $O$, et al. Prevalence and risk factors of lower reproductive tract infections in symptomatic women in Dakar, Senegal. Infect Dis 2019; 12: 1178633719851825, doi: $10.1177 / 1178633719851825$.

10. Moridi K, Hemmaty M, Azimian A, Fallah MH, Abyaneh HK, Ghazvini K. Epidemiology of genital infections caused by Mycoplasma hominis, M. genitalium and Ureaplasma urealyticum in Iran; a systematic review and meta-analysis study (2000-2019). BMC Public Health 2020; 20: 1020, doi: 10.1186/s12889-020-08962-5.

11. Tomić J, Ostojić M. The results of three-year monitoring of Mycoplasma hominis and Ureaplasma urealyticum in the cantons of Herzegovina-Neretva and West Herzegovina. Psychiatr Danub 2019; 31: 131-132.

12. Lee MY, Kim MH, Lee WI, Kang SY, Jeon YL. Prevalence and antibiotic susceptibility of Mycoplasma hominis and Ureaplasma urealyticum in pregnant women. Yonsei Med J 2016; 57: 1271-1275, doi: 10.3349/ymj.2016.57.5.1271.

13. Jang YS, Min JW, Kim YS. Positive culture rate and antimicrobial susceptibilities of Mycoplasma hominis and Ureaplasma urealyticum. Obstet Gynecol Sci 2019; 62: 127-133, doi: 10.5468/ogs.2019.62.2.127. 